\title{
Segmentation of Plaques in Sequences of Ultrasonic B-Mode Images of Carotid Arteries Based on Motion Estimation and a Bayesian Model
}

\author{
François Destrempes, Jean Meunier, Marie-France Giroux, Gilles Soulez, and Guy Cloutier*
}

\begin{abstract}
The goal of this paper is to perform a segmentation of atherosclerotic plaques in view of evaluating their burden and to provide boundaries for computing properties such as the plaque deformation and elasticity distribution (elastogram and modulogram). The echogenicity of a region of interest comprising the plaque, the vessel lumen, and the adventitia of the artery wall in an ultrasonic B-mode image was modeled by mixtures of three Nakagami distributions, which yielded the likelihood of a Bayesian segmentation model. The main contribution of this paper is the estimation of the motion field and its integration into the prior of the Bayesian model that included a local geometrical smoothness constraint, as well as an original spatiotemporal cohesion constraint. The Maximum A Posteriori of the proposed model was computed with a variant of the exploration/selection algorithm. The starting point is a manual segmentation of the first frame. The proposed method was quantitatively compared with manual segmentations of all frames by an expert technician. Various measures were used for this evaluation, including the mean point-to-point distance and the Hausdorff distance. Results were evaluated on 94 sequences of 33 patients (for a total of 8988 images). We report a mean pointto-point distance of $0.24 \pm 0.08 \mathrm{~mm}$ and a Hausdorff distance of $1.24 \pm 0.40 \mathrm{~mm}$. Our tests showed that the algorithm was not sensitive to the degree of stenosis or calcification.
\end{abstract}

Manuscript received July 28, 2010; revised December 1, 2010, February 14, 2011; accepted March 7, 2011. Date of publication March 14, 2011; date of current version July 20, 2011. This work was jointly supported by the Natural Sciences and Engineering Research Council of Canada under Grant 138570-06 and Grant 323405-06 and by the Canadian Institutes of Health Research under Grant PPP-78763. Asterisk indicates corresponding author

F. Destrempes is with the Laboratory of Biorheology and Medical Ultrasonics, University of Montreal Hospital Research Center (CRCHUM), Montréal, QC H2L-2W5, Canada (e-mail:francois.destrempes@crchum.qc.ca).

J. Meunier is with the Department of Computer Science and Operation Research (DIRO), University of Montreal, Montréal, QC H3C-3J7, Canada, and also with the Institute of Biomedical Engineering, University of Montreal, Montréal, QC H3T-1J4, Canada (e-mail: meunier@iro.umontreal.ca).

M.-F. Giroux is with the Department of Radiology, University of Montreal Hospital Center (CHUM), Montréal, QC H2L-4M1, Canada, and also with the Department of Radiology, Radio-Oncology, and Nuclear Medicine, University of Montreal, Montréal, QC H3T-1J4, Canada (e-mail: mfgiroux @ videotron.ca)

G. Soulez is with the Department of Radiology, University of Montreal Hospital Center (CHUM), Montréal, QC H2L-4M1, Canada, with the Department of Radiology, Radio-Oncology, and Nuclear Medicine, University of Montreal, Montréal, QC H3T-1J4, Canada, and also with the Institute of Biomedical Engineering, University of Montreal, Montréal, QC H3T-1J4, Canada (e-mail:gilles.soulez.chum@ssss.gouv.qc.ca).

*G. Cloutier is with the Laboratory of Biorheology and Medical Ultrasonics, University of Montreal Hospital Research Center (CRCHUM), Montréal, QC H2L-2W5, Canada, with the Department of Radiology, Radio-Oncology and Nuclear Medicine, University of Montreal, Montréal, QC H3T-1J4, Canada, and also with the Institute of Biomedical Engineering, University of Montreal, Montréal, QC H3T-1J4, Canada (e-mail:guy.cloutier@umontreal.ca).

Digital Object Identifier 10.1109/TBME.2011.2127476
Index Terms-B-Mode, Bayesian model, carotid artery, expectation maximization algorithm, exploration selection algorithm, mixtures of Nakagami distributions, motion estimation, plaque, segmentation, stochastic optimization, tracking, ultrasound.

\section{INTRODUCTION}

$\mathbf{P}$ RECISE segmentations of plaques in a sequence of ultrasonic B-mode images of the carotid artery allow the computation of various biomechanical and anatomical properties of the artery wall that may be useful to clinicians to follow the evolution of the atherosclerotic disease. In fact, in [1]-[4], an elasticity map is computed in a region of interest (ROI) of the artery wall along a few cardiac cycles to help determining the vulnerability of the plaque. Thus, it is useful to have a segmentation of the ROI through a whole sequence of ultrasound (US) images to track time-varying elasticity changes. Considering the amount of time required to perform manually the segmentation task as well as the intraobserver or interobserver variability that is usually associated with this task, an algorithm that performs the segmentation based on minimal user interaction is important in the research context.

Several algorithms for the segmentation of carotid arteries that present a plaque have been proposed in US imaging (see Table I). In [5] and [6], various snake segmentation methods, with initialization based on the blood flow image, were tested in the context of 2-D longitudinal images of carotid plaques. The plaque in 2-D longitudinal images is segmented in [7]-[9] using a combination of gradient-based segmentation, a snake segmentation method, and a fuzzy K-means algorithm, with an initialization based on pixel intensity. Hough transforms are used in [10] to perform the segmentation of 2-D longitudinal and cross-sectional images of plaques. Other papers (e.g. [11]-[15]) have presented atherosclerotic carotid segmentation techniques, but for the segmentation of the lumen rather than the plaque itself. The reader may consult [16]-[18] for numerous other references on US imaging, including intravascular ultrasound segmentation methods and tissue characterization.

In this paper, we present a method for the segmentation of plaques in video sequences of US images of the internal carotid artery (ICA). We formulate the proposed method in a Bayesian framework. The proposed Bayesian model is inspired by the model presented in [19]. As in [19], the likelihood of the proposed segmentation model is based on mixtures of Nakagami distributions, which are used to describe the statistical properties of the intensity in a B-mode image. The main addition to our previous paper [19], that was limited to healthy 
TABLE I

Overview of Atherosclerotic Carotid Plaque SEgmentation Techniques for Ultrasound Imaging

\begin{tabular}{|c||c|c|c|c|c|}
\hline Study & Segmentation Method & $\begin{array}{c}\text { Anatomy segmented, } \\
\text { geometrical view }\end{array}$ & $\begin{array}{c}\text { 2D/3D } \\
\text { temporal/still }\end{array}$ & $\begin{array}{c}\text { number of } \\
\text { sequences }\end{array}$ & $\begin{array}{c}\text { number of } \\
\text { images }\end{array}$ \\
\hline \hline Loizou [6] & $\begin{array}{c}\text { Snake segm. methods, with initialization } \\
\text { based on the blood flow image }\end{array}$ & $\begin{array}{c}\text { Plaque, } \\
\text { longitudinal }\end{array}$ & 2D, still & - & 80 \\
\hline Delsanto [8] & $\begin{array}{c}\text { Gradient-based segm., snake segm. } \\
\text { method, and fuzzy K-means alg., with } \\
\text { initialization based on pixel intensity }\end{array}$ & $\begin{array}{c}\text { IMT and plaque, } \\
\text { longitudinal }\end{array}$ & 2D, still & - & 64 (healthy) \\
& Hough transforms & $\begin{array}{c}\text { IMT and plaque, longitu- } \\
\text { dinal and cross-sectional }\end{array}$ & 2D, still & - & 10 (healthy) \\
& $\begin{array}{c}\text { Plaque, } \\
\text { longitudinal }\end{array}$ & 2D, temporal & 94 & 8988 \\
\hline Golemati [10] & $\begin{array}{c}\text { Stochastic optimization of a Bayesian } \\
\text { model, which is based on intensity distri- } \\
\text { butions and geometrical and spatio-temporal } \\
\text { priors that use motion estimation }\end{array}$ & & & \\
\hline
\end{tabular}

IMT is the intima-media thickness of the carotid wall.

carotids, is the consideration of more complex and moving echo textures of heterogeneous plaques, as well as variable shapes of the plaque. It includes a tracking method that uses an estimation of the motion field of the plaque in the video sequence based on optical flow estimates. The prediction of the position and shape of the plaque at the current frame (based on the motion field estimates) is then integrated directly into a spatiotemporal cohesion prior term of the proposed Bayesian model. We also consider an original geometrical local smoothness prior for the plaque boundaries. The segmentation problem is then formulated as the computation of the Maximum A Posteriori (MAP) of the Bayesian model. All computations are performed after the manual segmentation of the plaque in the first B-mode image of the video sequence (which is the only user interaction in the proposed method). Thus, the proposed method is semiautomatic. See [20] for a brief account of the proposed method.

The strategy of exploiting optical flow estimates to track motion has appeared in [21] and it was adopted in this study. In [21], it was used as an initialization of the velocities in an active contour segmentation method. In our case, the optical flow estimates are based on a first-order tissue dynamics model (i.e., motion field), which simplifies their computation. We show that for the database used in the reported tests, the hypothesis of optical flow is self-consistent, due mainly to small deformations of the plaque from one frame to the next one. Moreover, in our case, the optical flow estimates are integrated into the prior of a region-based segmentation model, rather than a contour-based segmentation model (as in [21]).

The proposed method was tested on 94 sequences of 2-D longitudinal images of internal carotid arteries of 33 patients, for a total of 8988 images. The size of our database compares very favorably with previous studies [6] (80 images), [8] (56 images), and [10] (4 images) that are also testing a segmentation method of longitudinal images of plaques. Additional material including a segmented video sequence can be found at our website http://www.lbum-crchum.com (see the publication section).

\section{MATERIALS}

\section{A. Image Acquisition}

The RF images were acquired with a $10-\mathrm{MHz} 38-\mathrm{mm}$ linear array transducer of a Sonix RP echograph (Ultrasonix,
Vancouver, BC, Canada). The frame rate was set by the radiologist and depended on the video sequence. It varied between 19 and $24 \mathrm{~Hz}$, and the average duration of the sequences acquired over consecutive cardiac cycles was about $7 \mathrm{~s}$. For 74 of the video sequences used in the tests reported in Section VI, $1 \mathrm{~mm}$ corresponds to 51.9 pixels in the axial direction, whereas in the lateral direction, $1 \mathrm{~mm}$ is equal to about 6.7 pixels (i.e., 256 scan lines for $38 \mathrm{~mm}$ ). For the other 20 sequences, the axial resolution had been changed at the acquisition step, and $1 \mathrm{~mm}$ corresponds to about 26 pixels in the axial direction (due to a new version of the Sonix RP echograph software).

\section{B. Data}

We considered 94 video sequences of B-mode images (i.e., the uncompressed envelope of the acquired RF images) from 33 patients with carotid plaques. Women and men of 40 years or older for which at least one of the carotid arteries presented a stenosis of at least $50 \%$ (in diameter reduction) were included in our study. Pathological subjects for whom carotid atherosclerosis was newly diagnosed were recruited by vascular radiologists, neuroradiologists or neurologists of the University of Montreal Hospital Center (CHUM) and by the service of Vascular Neurology of CHUM. The Institutional Review Board approved this health insurance portability and accountability act-compliant research project, and patients signed an informed consent. There were 22 men and 11 women with a mean age of $71.5 \pm 7.5$ years. Their body mass index was $25.7 \pm 3.4 \mathrm{Kg} / \mathrm{m}^{2}$. For most patients, longitudinal views of the left and right proximal internal carotid arteries were acquired independently by two expert radiologists, for a total of four video sequences per patient. However, in some cases, only the left or right proximal internal carotid was acquired (seven patients), so that the total number of carotid arteries was actually 59. Some of the sequences were not acquired on both sides due to the presence of a thrombosis or a past surgery on the carotid artery. Also, in some cases, only one of the expert radiologists performed an acquisition (on 24 carotid arteries). The degrees of stenosis and calcification [22] for the 94 sequences are indicated in Table II. When available, stenoses were graded on angiography by computed tomography (CT), magnetic resonance (MR) or conventional angiography, using North American Symptomatic Carotid Endarterectomy Trial (NASCET) criteria [23]. If MR, CT, or catheter 
TABLE II

DEGREe of PlaQue StenOSIS IN DiAMETER REDUCTION AND CALCIFICATION FOR THE 94 SEQUENCES OF B-MODE IMAGES IN THE DATABASE USED FOR THIS STUDY

\begin{tabular}{|l||c|c|c|c||c|}
\hline \multicolumn{1}{|c||}{} & \multicolumn{5}{c||}{ degree of stenosis } \\
\hline calcification & $0-30 \%$ & $30-50 \%$ & $50-70 \%$ & $>70 \%$ & total \\
\hline \hline none & 8 & 1 & 6 & 4 & 19 \\
\hline mild & 13 & 1 & 2 & 6 & 22 \\
\hline moderate & 9 & 3 & 11 & 12 & 35 \\
\hline severe & 1 & 7 & 1 & 5 & 14 \\
\hline $\begin{array}{l}\text { missing } \\
\text { information }\end{array}$ & 0 & 1 & 2 & 1 & 4 \\
\hline \hline total & 31 & 13 & 22 & 28 & 94 \\
\hline
\end{tabular}

angiography were not available, the degree of carotid stenosis was determined on Doppler ultrasound examination, using the following criteria [22]: 1) $<30 \%$ when the ICA peak systolic velocity (PSV) was less than $125 \mathrm{~cm} / \mathrm{s}$ and no or minimal (less than $30 \%$ in B-mode) plaque or intimal thickening was visible; 2) 30\%-50\% stenosis when ICA PSV was less than 125 $\mathrm{cm} / \mathrm{s}$ and a plaque or an intimal thickening between $30 \%$ and $50 \%$ was visible on B-mode; 3 ) $50 \%-70 \%$ stenosis when ICA PSV was $125-230 \mathrm{~cm} / \mathrm{s}$ and a plaque was visible on B-mode; 4) $>70 \%$ stenosis to near occlusion when ICA PSV was greater than $230 \mathrm{~cm} / \mathrm{s}$ and a visible plaque and a lumen narrowing were seen; 5) $95 \%$ for near occlusion when there was a markedly narrowed lumen on color Doppler US; and 6) 100\% or total occlusion when there was no detectable patent lumen on grayscale US and no flow on spectral power, and color Doppler US. Note that a $100 \%$ stenosis or an occlusion was not included in our database.

\section{TRacking the Position AND ShaPe OF the PlaQue USING MOTION VECTOR FIELDS}

\section{A. Motion Field}

Given a sequence of $T$ RF images, $r(x, y, t)$ denotes the brightness of the B-mode envelope of the RF signal in the $t$ th frame at pixel $(x, y)$, where $x$ and $y$ denote the lateral and axial directions, respectively. Similarly to [24], we adopted the following first-order tissue dynamics:

$$
(x, y) \mapsto \tau+\mathcal{L}(x, y)
$$

where

$$
\tau=\left(\begin{array}{c}
\tau_{x} \\
\tau_{y}
\end{array}\right) ; \quad \mathcal{L}(x, y)=\left(\begin{array}{cc}
\delta_{11}+1 & \delta_{12} \\
\delta_{21} & \delta_{22}+1
\end{array}\right)\left(\begin{array}{l}
x \\
y
\end{array}\right) .
$$

Concretely, this means that a point of the plaque or its surrounding tissues with coordinates $(x, y)$ at frame $t$ gets mapped to the point with coordinates $\tau+\mathcal{L}(x, y)$ at frame $t+1$. In particular, $\tau+\mathcal{L}$ can be viewed as a motion field of the plaque and its surrounding tissues, where $\tau$ denotes a translation and $\mathcal{L}$ a deformation.

In Section III-B, we propose the use of optical flow to estimate the motion field $\tau+\mathcal{L}$. Thus, one is interested in the correlation between the gray level of the B-mode image $r(x, y, t)$ at frame $t$ and the registered B-mode image $r\left(x_{*}, y_{*}, t+1\right)$, where $\left(x_{*}, y_{*}\right)=(\tau+\mathcal{L})(x, y)$, i.e., the B-mode envelope of the Lagrangian speckle image of [25]. The optical flow hy- pothesis is equivalent to a perfect correlation between the two images $r(x, y, t)$ and $r\left(x_{*}, y_{*}, t+1\right)$, and the conservation of their mean gray level (MGL).

We present in Section VII-B empirical tests for verifying the hypotheses of the correlation between the B-mode images and the conservation of their MGL from one frame to the next, in the case of our database. The outcome is that the use of optical flow is self-consistent on our database.

\section{B. Estimating the Motion Field}

As discussed in Section III-A, we assumed only small deformations, and this hypothesis was verified on the database as reported in Section VII-B. In other words, we assumed the conservation of the B-mode gray level over time: $r(x, y, t) \equiv$ constant, $t \geq 1$, which yields the well-known identity of the optical flow

$$
\begin{aligned}
& \frac{\partial}{\partial x} r(x, y, t) d x+\frac{\partial}{\partial y} r(x, y, t) d y \\
& \quad=-\frac{\partial}{\partial t} r(x, y, t) d t .
\end{aligned}
$$

Taking $d t=1$ (corresponding to frames $t$ and $t+1$ ), under the tissue dynamics of (1), we have

$$
\left(\begin{array}{l}
d x \\
d y
\end{array}\right)=\tau+\mathcal{L}(x, y)-\left(\begin{array}{l}
x \\
y
\end{array}\right)=\tau+\left(\begin{array}{ll}
\delta_{11} & \delta_{12} \\
\delta_{21} & \delta_{22}
\end{array}\right)\left(\begin{array}{l}
x \\
y
\end{array}\right) .
$$

Thus, given a segmentation $R$ of the plaque at the $t$ th frame, each pixel $(x, y)$ within a neighborhood of the segmented region gives rise to the linear equation

$$
\begin{aligned}
& \frac{\partial}{\partial x} r(x, y, t)\left(\tau_{x}+\delta_{11} x+\delta_{12} y\right) \\
& \quad+\frac{\partial}{\partial y} r(x, y, t)\left(\tau_{y}+\delta_{21} x+\delta_{22} y\right)=-\frac{\partial}{\partial t} r(x, y, t)
\end{aligned}
$$

in the six variables $\tau_{x}, \delta_{11}, \delta_{12}, \tau_{y}, \delta_{21}$, and $\delta_{22}$. In our application, the number of points (and hence of equations) is greater than 6 , and therefore, we obtained an overdetermined system of linear equations. This system of equations (and hence, the motion field) was then estimated in the sense of the least mean square (LMS). In our implementation, we took as neighborhood, the region encompassed by the set of points within $2 \mathrm{~mm}$ of the segmented region $R$. Considering a neighborhood of the plaque allows us to take into account the motion field at the boundary of the plaque and slightly beyond, rather than only in its interior.

For the computation of the partial derivatives in (5), we first applied a uniform low-pass spatial filter of size $0.75 \times$ $0.25 \mathrm{~mm}^{2}$ (laterally, axially) ${ }^{1}$ to the B-mode image, resulting in an image $\bar{r}(x, y, t)$. We then made use of the following secondorder approximations:

$$
\begin{aligned}
& \frac{\partial}{\partial x} r(x, y, t) \\
& \quad \approx \frac{1}{8}(\bar{r}(x+1, y-1, t-1)-\bar{r}(x-1, y-1, t-1)
\end{aligned}
$$

\footnotetext{
${ }^{1}$ That is $5 \times 13$ pixels $^{2}$ for the first 74 video sequences and $5 \times 7$ pixels $^{2}$ for the 20 remaining ones.
} 


$$
\begin{aligned}
& +\bar{r}(x+1, y+1, t-1)-\bar{r}(x-1, y+1, t-1) \\
& +\bar{r}(x+1, y-1, t+1)-\bar{r}(x-1, y-1, t+1) \\
& +\bar{r}(x+1, y+1, t+1)-\bar{r}(x-1, y+1, t+1))
\end{aligned}
$$

and similarly for $\frac{\partial}{\partial y} r(x, y, t)$ and $\frac{\partial}{\partial t} r(x, y, t)$. At the boundaries of the video sequences (i.e., $t=1$ or $t=T$ ) or at the boundaries of the images, the second-order approximations of (6) were adjusted accordingly, making use of first-order approximations if necessary. For instance, if $t=1$ and $x$ coincides with the left boundary of the image, we used the first-order approximation

$$
\begin{aligned}
& \frac{\partial}{\partial x} r(x, y, t) \\
& \quad \approx \frac{1}{4}(\bar{r}(x+1, y-1, t)-\bar{r}(x, y-1, t) \\
& \quad+\bar{r}(x+1, y+1, t)-\bar{r}(x, y+1, t) \\
& \quad+\bar{r}(x+1, y-1, t+1)-\bar{r}(x, y-1, t+1) \\
& \quad+\bar{r}(x+1, y+1, t+1)-\bar{r}(x, y+1, t+1)) .
\end{aligned}
$$

\section{Propagation of a Segmented Region Through}

Successive Motion Fields

Given two frames, $t^{\prime}<t$, let $R$ be a segmented region at frame $t^{\prime}$. Then, the motion fields $(\tau+\mathcal{L})^{t^{\prime}}, \ldots,(\tau+\mathcal{L})^{t-1}$ were learned recursively as follows, in our implementation. The motion field $(\tau+\mathcal{L})^{t^{\prime}}$ was estimated on a neighborhood of the segmented region $R$ at frame $t^{\prime}$, as explained in Section III-B; applying that motion field pointwise [using (1) and (2)] to the boundary of the region $R$ yielded a new region $R^{\prime}$ at frame $t^{\prime}+1$; then, the motion field at the next frame $t^{\prime}+1$ was learned on a neighborhood of $R^{\prime}$, and so on. Combining these motion fields for frames $t^{\prime}$ up to $t-1$, in that order, yielded the propagation of the region $R$ in frame $t^{\prime}$ to a region in frame $t$.

\section{Average of Propagations of Segmented Regions at Previous Frames}

At the first frame, we took the manual segmentation of the plaque as initial segmentation. At an ulterior frame $t>1$, one could take the segmentation of the plaque at the previous frame $t-1$ and use its propagation at frame $t$ (using the propagation method of Section III-C) as a prediction of the segmentation at frame $t$. For more robustness, we considered the various segmentations of the plaque at previous frames, and each of these segmentations was propagated at frame $t$ according to the method of Section III-C. We then considered the average of these propagations as a prediction of the segmentation at frame $t$. In our implementation, we considered the immediate previous frames, corresponding to about $1 \mathrm{~s}$ of the video sequence.

\section{Segmentation of the Plaque Using A PREDICTION OF THE SOLUTION}

In this section, we present a refinement of the Bayesian model introduced in [19] that takes into account the great variability in the shape of a plaque, as well as its displacement [26] and deformation [1] from one frame to the next. Based on the pre-

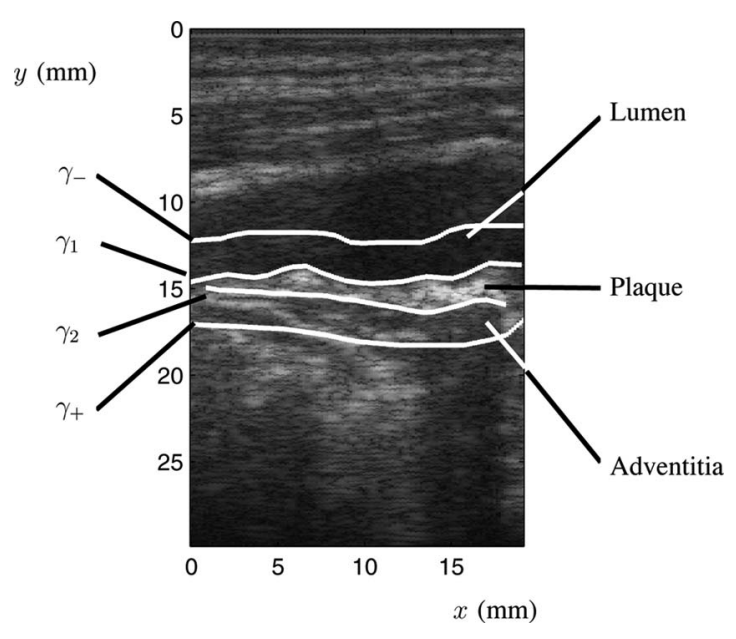

Fig. 1. Curves appearing in the figure are from top to bottom, $\gamma_{-}, \gamma_{1}, \gamma_{2}$, and $\gamma_{+}$. The curves $\gamma_{1}$ and $\gamma_{2}$ delimiting the IMT and the plaque are constrained within the region between $\gamma_{-}$and $\gamma_{+}$. The region between $\gamma_{-}$and $\gamma_{1}$ consists mainly of the lumen; the region between $\gamma_{2}$ and $\gamma_{+}$consists mainly of the surrounding tissues (including the adventitia). The solution $\left(\gamma_{1}, \gamma_{2}\right)$ of the proposed method is viewed as the MAP of a Bayesian model (see Section IV-D).

diction of the segmented plaque described in Section III-D, one considers the following Bayesian model.

\section{A. Random Fields}

Given $r(x, y, t)$ the gray level of the B-mode image in the $t$ th frame at pixel $(x, y), I(x, y, t)=r(x, y, t)^{2}$ is called the intensity. The field $I^{(t)}=(I(x, y, t))$ is viewed as the observable random field. We converted here the gray level $r$ of the Bmode image into its intensity $I=r^{2}$ for convenience. Namely, a Nakagami distribution on the variable $r$ is equivalent to the simpler gamma distribution on the intensity $I=r^{2}$.

Consider a ROI that includes the plaque in the carotid wall on a longitudinal (lateral) view, some portion of the lumen, and the adventitia, and let the ROI be delimited by two curves $\gamma_{-}^{(t)}$ and $\gamma_{+}^{(t)}$, where the first one is within the lumen and the second one outside the carotid wall. We wish to determine the curves $\gamma_{1}^{(t)}$ and $\gamma_{2}^{(t)}$ representing the interface between the plaque and the lumen or the adventitia, respectively, in the $t$ th frame. Note that these two interface curves are located within the ROI (see Fig. 1).

\section{B. Likelihood of the Segmentation Model}

The intensity of each tissue (or layers of tissues) was characterized by a mixture of three gamma distributions, with proportions specific to the tissue: $f_{j}(I)=\sum_{i=1}^{3} q_{i}^{j} \mathcal{G}\left(I \mid k_{i}, \theta_{i}\right)$, where $j=1,2,3$ corresponds, respectively, to the lumen, the plaque, and the adventitia of the carotid artery. Here, the weights satisfy $\sum_{i=1}^{3} q_{i}^{j}=1$, for $j=1,2,3$, as well as $q_{i}^{j} \geq 0$, for $i, j,=1,2,3$. Recall that the gamma distribution $\mathcal{G}(I \mid k, \theta)$ is defined by $\frac{I^{k-1}}{\Gamma(k) \theta^{k}} e^{-\frac{I}{\theta}}$ (where the symbol $\Gamma$ stands for the Euler gamma function). Note, as in [19], that a gamma distribution on the intensity $I$ is equivalent to a Nakagami distribution on the amplitude $\sqrt{I}$. For each tissue $j=1,2,3$ (i.e., lumen, plaque, or 
adventitia), the three coefficients $q_{i}^{j}, i=1,2,3$, were estimated using a straightforward expectation-maximization (EM) algorithm [27]. This latter point is the only difference with [19], as far as the likelihood is concerned. In fact, due to the hypothesis of a healthy carotid artery, the lumen had been matched with the gamma distribution having the lowest mean intensity, the adventitia corresponded to the gamma distribution having the highest mean intensity, whereas the IMT corresponded to a mixture of the three gamma distributions. Details on the likelihood can be found in [19] and [20].

Now, let the pair of curves $\left(\gamma_{1}^{(t)}, \gamma_{2}^{(t)}\right)$ represent the interface between the lumen and the plaque or the plaque and the adventitia, respectively. Using the identification of each tissue to a mixture of distributions, the likelihood $P_{t}\left(I^{(t)} \mid \gamma_{1}^{(t)}, \gamma_{2}^{(t)}\right)$ of the segmentation model was defined as in [19]

$$
\prod_{s \in R_{1}} f_{1}^{(t)}\left(I_{s}^{(t)}\right) \times \prod_{s \in R_{2}} f_{2}^{(t)}\left(I_{s}^{(t)}\right) \times \prod_{s \in R_{3}} f_{3}^{(t)}\left(I_{s}^{(t)}\right)
$$

where $R_{1}$ is the region delimited by the pair of curves $\left(\gamma_{-}^{(t)}, \gamma_{1}^{(t)}\right)$ (the lumen), $R_{2}$ is the region delimited by $\left(\gamma_{1}^{(t)}, \gamma_{2}^{(t)}\right)$ (the plaque), and $R_{3}$ is the region delimited by $\left(\gamma_{2}^{(t)}, \gamma_{+}^{(t)}\right)$ (the adventitia).

\section{Prior of the Segmentation Model}

In the case of healthy carotids, anatomical information could be used to construct a prior for the segmentation model (see [19]), namely, the average IMT, as well as its spatial and temporal variances (in the sense of [28]). However, these values cover only the expected dimensions of healthy carotids. As we are interested in patients presenting pathologies of the carotid wall in this paper, a prior based on a manual segmentation of the plaque in the first frame was used instead. Namely, two constraints that regularize the segmentation process were considered. These constraints differ from the ones presented in [19].

1) Geometrical Smoothness Constraint: Let $\gamma$ be a continuous piecewise linear curve of the form $\gamma(l)=(x(l), y(l))$, for $l \in[1, L]$, with vertices $(x(1), y(1)), \ldots,(x(L), y(L))$, where the first coordinate indicates the lateral position and the second, the axial position. To take into account the different scaling along the axial and lateral directions, we considered the ratio $\rho=51.9 / 6.7$ ( 1 pixel in the lateral direction corresponds to $\rho \approx 7.7$ pixels in the axial direction), or $\rho=26 / 6.7 \approx 3.9$ (depending on the axial resolution). Accordingly, we considered the curve $\gamma_{\rho}(l)=(\rho x(l), y(l))$. Doing so yields the ratio between the lateral and axial coordinates in the real scene (in millimeter), rather than the ratio of the coordinates in the image (in pixels). For simplicity of notation, we drop the subindex $\rho$ in what follows, so that $\gamma$ now stands for $\gamma_{\rho}$.

A geometrical smoothing constraint $\Delta_{\mathrm{sm}, d}(\gamma)$ was then defined as the line integral

$$
\rho \int_{\gamma} G_{d} d \gamma=\rho \int_{1}^{L} G_{d}(\gamma(l))\|\dot{\gamma}(l)\| d l
$$

where $\dot{\gamma}(l)$ denotes the derivative of the curve $\gamma(l)$, and $G_{d}(\gamma(l))$ is a local measure of the abruptness of the curve $\gamma$ in a neigh- borhood of the given point $\gamma(l)$ (see below for additional information). If $\gamma$ is rectilinear at the given point, then $G_{d}$ has a value of 0 , and the more the $\gamma$ is abrupt at the given point, the higher is the value of $G_{d}$. The parameter $d$ controls the size of the local neighborhood.

Now, consider the direction vector of the tangent vector to the curve $\gamma$ (i.e., $\left(\frac{\dot{x}}{\sqrt{\dot{x}^{2}+\dot{y}^{2}}}, \frac{\dot{y}}{\sqrt{\dot{x}^{2}+\dot{y}^{2}}}\right)$, where the "dot" denotes the derivative). Then, a natural choice of measure of abruptness is the variance of the coordinates of that direction vector in a neighborhood of radius $d$ along the curve and centered at the given point $\gamma(l)$, i.e., $G_{d}(\gamma(l))=\operatorname{Var}\left[\frac{\dot{x}}{\sqrt{\dot{x}^{2}+\dot{y}^{2}}}\right]+\operatorname{Var}\left[\frac{\dot{y}}{\sqrt{\dot{x}^{2}+\dot{y}^{2}}}\right]$. If $d$ covers the entire curve, an overall rectilinear shape of the plaque (or the IMT) is expected. Since we consider the presence of vascular pathologies, we selected a smaller value; namely, $d$ corresponds to 5 pixels $(0.75 \mathrm{~mm})$ in the lateral direction, thus allowing a greater variability in the shape of the plaque, since the constraint is more local.

2) Spatiotemporal Cohesion Constraint: Let $\left(\gamma_{1, \mathrm{pred}}\right.$, $\left.\gamma_{2, \text { pred }}\right)$ be a predicted solution and let $\left(\gamma_{1}, \gamma_{2}\right)$ be a pair of curves (we drop the index $(t)$ in this paragraph). In this paper, we used the propagations of solutions in previous frames (cf., Section III-D) as a predicted solution, in order to take into account the lateral and axial displacements of the plaque and its variable shape. Preliminary tests on a few video sequences of plaques convinced us that it was a better strategy than using only the solution in the previous frame. A spatiotemporal cohesion constraint at frame $t$ encourages the curve $\gamma_{i}$ to be near $\gamma_{i, \text { pred }}$. Namely, we defined the constraint $\Delta_{\mathrm{tm}}\left(\gamma_{i}, \gamma_{i, \text { pred }}\right)$ as

$$
\frac{1}{2 \sigma_{t}^{2}} \int_{1}^{L}\left\|\gamma_{i}(l)-\gamma_{i, \text { pred }}(l)\right\|^{2}\left(\left\|\dot{\gamma}_{i}(l)\right\|+\left\|\dot{\gamma}_{i, \text { pred }}(l)\right\|\right) / 2 d l
$$

where the standard deviation is taken equal to $\sigma_{t}=0.12 \mathrm{~mm}$ (i.e., the temporal standard deviation of the IMT as in [28]). The function $\Delta_{\mathrm{tm}}\left(\gamma_{i}, \gamma_{i, \text { pred }}\right)$ is equal to 0 if and only if $\gamma_{i}=\gamma_{i, \text { pred }}$, and it is symmetric. We used it as a measure of the discrepancy between the segmented curve and the predicted solution.

Altogether, combining the two constraints mentioned earlier, we considered the prior $P_{t}\left(\gamma_{1}^{(t)}, \gamma_{2}^{(t)} \mid \gamma_{1, \text { pred }}^{(t)}, \gamma_{2 \text {,pred }}^{(t)}\right)$ proportional to

$$
\prod_{i=1}^{2} \exp \left(-\beta \Delta_{\mathrm{sm}, d}\left(\gamma_{i}^{(t)}\right)-\Delta_{\mathrm{tm}}\left(\gamma_{i}^{(t)}, \gamma_{i, \mathrm{pred}}^{(t)}\right)\right)
$$

for $t>1$, where $\beta>0$ is the weight of the geometrical smoothing constraint. The value of $\beta$ was estimated, based on the manual segmentation of the first frame, in the sense of the Maximum Pseudo-A-Posteriori estimator (that is closely related to the Maximum Pseudo-Likelihood estimator of [29], [30]). See the Appendix for details. In our tests, the estimated value of $\beta$ varied between 0.2 and 10 .

\section{Posterior Distribution of the Segmentation Model}

Combining the likelihood of (8) and the prior of (11), one obtains the following expression for the posterior distribution $P_{t}\left(\gamma_{1}^{(t)}, \gamma_{2}^{(t)} \mid I^{(t)}, \gamma_{1, \text { pred }}^{(t)}, \gamma_{2, \text { pred }}^{(t)}\right)$ of the proposed 
segmentation model up to a normalizing constant:

$$
P_{t}\left(I^{(t)} \mid \gamma_{1}^{(t)}, \gamma_{2}^{(t)}\right) P_{t}\left(\gamma_{1}^{(t)}, \gamma_{2}^{(t)} \mid \gamma_{1, \text { pred }}^{(t)}, \gamma_{2, \text { pred }}^{(t)}\right)
$$

The proposed method consisted in estimating $\left(\gamma_{1}^{(t)}, \gamma_{2}^{(t)}\right)$ in the sense of the MAP, i.e., to find $\left(\gamma_{1}^{(t)}, \gamma_{2}^{(t)}\right)$ that maximizes (12).

The MAP solution was computed using the stochastic optimization algorithm exploration/selection (ES) of [19], with an adaptation to the data structure that takes into account the variable shapes of plaques.

\section{Comparative Manual Assessment}

Let $\mathcal{M}\left(R, R_{\text {man }}\right)$ be a measure of agreement or, on the contrary, a measure of discrepancy between two (segmented) regions $R$ and $R_{\text {man }}$. Then, if $\left(R^{(t)}\right)$ and $\left(R_{\operatorname{man}}^{(t)}\right), t=1, \ldots, T$, are the sequences of regions corresponding to the semiautomatic segmentation and the manual segmentation by an expert technician, respectively, for a same video sequence (of $T$ frames), one can define a measure between the two segmentations upon averaging the measure over all frames of that sequence: $\mathcal{M}=\frac{1}{T} \sum_{t=1}^{T} \mathcal{M}\left(R^{(t)}, R_{\text {man }}^{(t)}\right)$. Note that $R_{\text {man }}^{(1)}$ is the manual segmentation in the first frame that was used as initialization of the proposed segmentation algorithm. The manual segmentations in the other frames were used solely for the validation of the method, as described in this section.

In our tests, we considered the following four measures of agreement based on receiver operating characteristic analysis [31], [32] between a semiautomatic segmentation $R$ and a manual segmentation $R_{\text {man }}$. The four measures are the sensitivity (also called true positive rate or recall), the specificity, the kappa index (also called the F-measure), and the overlap which are respectively defined as follows:

$$
\begin{aligned}
& \mathrm{se}=\frac{\left|R \cap R_{\mathrm{man}}\right|}{\left|R_{\mathrm{man}}\right|} ; \quad \mathrm{sp}=\frac{\left|\bar{R} \cap \overline{R_{\mathrm{man}}}\right|}{\left|\overline{R_{\text {man }}}\right|} ; \\
& \mathrm{KI}=2 \frac{\left|R \cap R_{\mathrm{man}}\right|}{|R|+\left|R_{\mathrm{man}}\right|} ; \quad \text { ov }=\frac{\left|R \cap R_{\mathrm{man}}\right|}{\left|R \cup R_{\mathrm{man}}\right|} .
\end{aligned}
$$

These four measures (together with the precision and the accuracy) were used in the study [6] ( $R$ and $R_{\text {man }}$ correspond to "AS" and "GT", respectively, in [6, eq. (1)]. Since the precision and the accuracy are redundant with the other four measures, we do not report them in this paper. Note that the measure of specificity requires the notion of the complement of the segmented region, which in turn depends arbitrarily on the ROI chosen to define the complement. For instance, if the ROI is enlarged, then the false positive rate decreases artificially. For that reason, we took the smallest rectangle (parallel to the axial and lateral axes) that contained both $R$ and $R_{\text {man }}$ (see Fig. 2 for an illustration).

We also considered four measures of discrepancy. The first one is the error of area in [12], and is defined as follows:

$$
\text { EArea }=\frac{|R|-\left|R_{\text {man }}\right|}{\left|R_{\text {man }}\right|} .
$$

The sign indicates whether the area is overestimated (+) or underestimated (-). We also computed the absolute error of

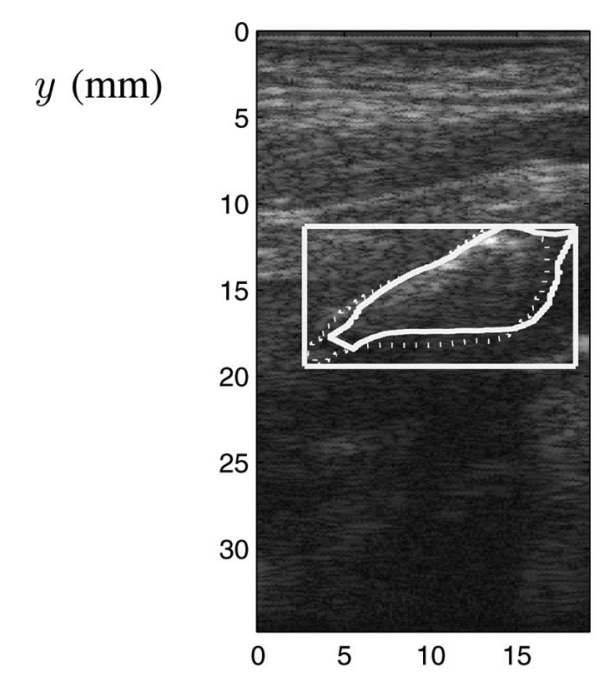

$x(\mathrm{~mm})$

Fig. 2. Two segmented regions and the smallest rectangle parallel to the axial and lateral axes that contains both of them. The dotted line: manual segmentation and the full line: semiautomatic segmentation.

area defined by

$$
\text { AEArea }=\frac{\left\|R|-| R_{\text {man }}\right\|}{\left|R_{\text {man }}\right|} .
$$

The two other measures of discrepancy are based on the average point-to-point distance $D$ and the Hausdorff pointto-point distance HD, respectively (cf., [33]), between the boundary of the region $R$ and that of the region $R_{\text {man }}$. Recall that the Hausdorff distance between two sets $A$ and $B$ is defined as $\operatorname{HD}(A, B)=\max (h(A, B), h(B, A))$, where $h(A, B)=\max _{a \in A} \min _{b \in B}\|a-b\|(\|a-b\|$ is the Euclidean norm). If $d$ is the Hausdorff distance between two sets $A$ and $B$, then every point of $A$ must be within a distance $d$ of $B$ and vice versa (cf., [33]).

\section{EXPERIMENTAL RESULTS}

The manual segmentations were performed by one expert technician and reviewed by a vascular sonographer for each frame of the 94 sequences. In our tests, the expert technician chose the temporal interval of each sequence where good quality images were present and with minimal transient motion artifacts due to movements of the transducer or of the patient, and performed the manual segmentations of the plaque throughout the chosen interval. The expert technician used a software developed in our laboratory to perform the manual segmentations. Once the sequence was loaded, a video of the full sequence could be viewed. The manual segmentation done in a frame could be copied on the next frame, and then adjusted through control points of a spline curve, if necessary. In total, 8988 frames were manually segmented. Fig. 3 shows four examples of semiautomatic segmentations of plaques.

Whenever the plaque was located on both the near and the far carotid artery wall (this fact holds for 36 of the 94 sequences), thus comprising two distinct connected components (see the second and fourth examples of Fig. 3), we used the segmentation method independently on the two components (each one with 

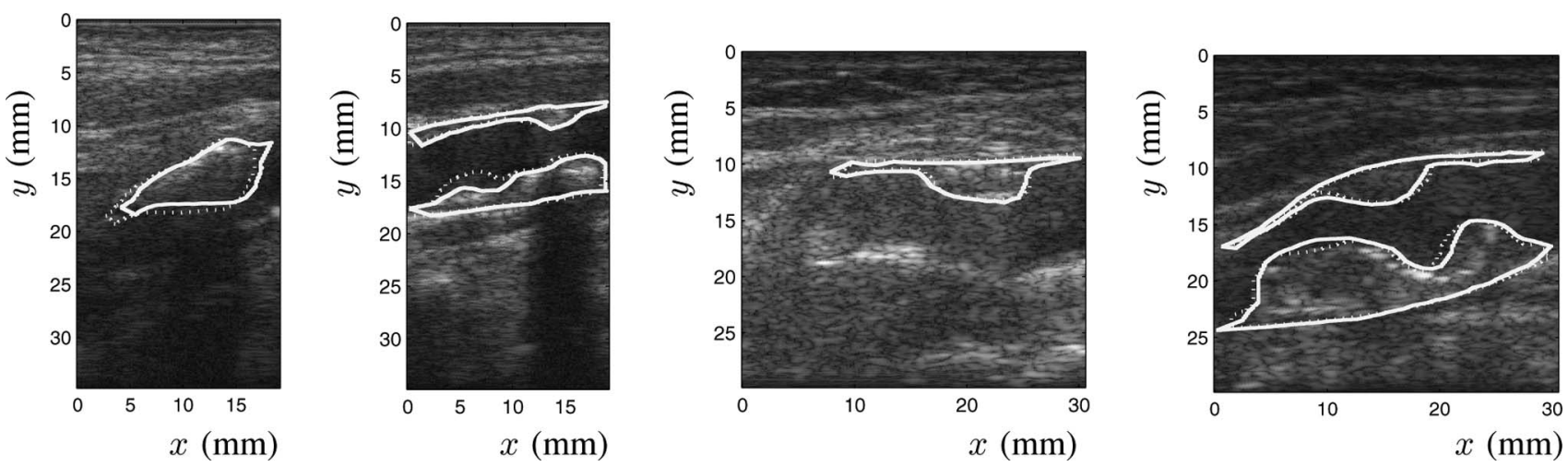

Fig. 3. Examples of semiautomatic (full line) and manual (dotted line) segmentations of plaques appearing at the proximal internal carotid, at the 60th frame of the segmented sequence. Two leftmost images: left and right carotids of a patient acquired by a same operator. Two rightmost images: left and right carotids of another patient acquired by a same operator.

TABLE III

QuANTItative EVALUATION OF THE SEGMENTATIONS OBTAINED By THE Proposed Method, Using Manual Segmentations Performed by AN EXPERT TECHNICIAN AS GROUND TRUTH

\begin{tabular}{|c||c|c|c|c|}
\hline Study & se (\%) & sp (\%) & KI (\%) & ov (\%) \\
\hline \hline Loizou [6] $(N=80)$ & 81.8 & 93.5 & 78.9 & 67.6 \\
& 80.4 & 94.6 & 77.9 & 67.8 \\
& 82.7 & 94.2 & 80.7 & 69.3 \\
& 79.6 & 93.7 & 77.3 & 66.6 \\
\hline Golemati [10] $(N=4)$ & $97.5 \pm 1.0$ & $95.5 \pm 1.0$ & - & - \\
& $95.0 \pm 4.1$ & $97.3 \pm 2.4$ & - & - \\
\hline Present study $(N=8988)$ & $83.7 \pm 8.3$ & $94.1 \pm 4.2$ & $84.8 \pm 7.5$ & $74.6 \pm 10.1$ \\
\hline
\end{tabular}

Four measures of agreement are reported: the sensitivity (se), the specificity (sp), the kappa index (KI), and the overlap (ov).

Measures are averaged over all frames of a sequence. see Section $\mathrm{V}$ for further details. The results are compared with the studies [6], [10].

In [6], there are four variants of the method based on different snake segmentation algorithms (corresponding to the four rows in this table).

In [10], there are two values corresponding to the systole and the diastole.

TABLE IV

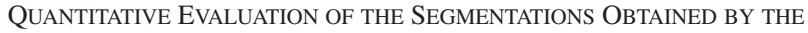
PROPOSED METHOD, Using MANUAL SEgmentations PERFORMED By AN EXPERT TECHNICIAN FOR THE ASSESSMENT

\begin{tabular}{|c||c|c|c|c|}
\hline & $\begin{array}{c}\text { EArea (\%) } \\
\text { (plaque) }\end{array}$ & $\begin{array}{c}\text { AEArea (\%) } \\
\text { (plaque) }\end{array}$ & D (mm) & HD (mm) \\
\hline \hline Present study & $-2.52 \pm 7.97$ & $7.07 \pm 4.99$ & $0.24 \pm 0.08$ & $1.24 \pm 0.40$ \\
\hline
\end{tabular}

Four measures of discrepancy are reported: the error of area (EA rea), the absolute error of area (AEA rea), the mean distance $(D)$, and the Hausdorff distance (HD).

Measures are averaged over all frames of a sequence. See Section $V$ for further details

its own manual initialization). Henceforth, there were actually a total of 130 video-sequence segmentations to be performed using the proposed algorithm. This represented 12206 frame segmentations, but the total number of images was 8988 .

The quantitative results of the evaluation method are presented in Tables III and IV. Namely, the measures of agreement or discrepancy of Section V, between the algorithmic and manual segmentations, were computed. In these tables, we included results from [6] and [10] that are discussed in Section VII-A.

The EM algorithm combined with the segmentation method took $129 \mathrm{~h}$ and $41 \mathrm{~min}$ (this represents 63391 estimations and 12206 frame segmentations) for the 130 video sequences. Thus, segmenting one frame took $38 \mathrm{~s}$ on average. The implementation was in $\mathrm{C}++$ and the tests were run on a $3 \mathrm{GHz}$ Pentium $4 \mathrm{CPU}$.

\section{DISCUSSION}

\section{A. Comparison of the Segmentation Method With Other Studies}

The results of Table III indicate that the proposed method compares favorably with [6], especially for the kappa index and the overlap measures. Note, however, that no standard deviation of the measures was reported in that study. On the other hand, the results reported in [10] are better than the ones obtained in [6] and this study. But then, the database size was very small (4 plaques) in [10].

Comparing with our previous study [19], we see in Table IV that the mean distance $(0.24 \pm 0.08 \mathrm{~mm})$ between the semiautomatic and the manual segmentations is smaller than the mean distance between the segmentations of the intima-media layers of disease-free proximal internal carotid arteries performed by two expert technicians (the mean distance was $0.33 \pm 0.17 \mathrm{~mm}$ for the lumen-intima interface and it was $0.40 \pm 0.29 \mathrm{~mm}$ for the media-adventitia interface). However, the Hausdorff distance was better in [19] $(0.73 \pm 0.33 \mathrm{~mm}$ for the lumen-intima interface and $0.88 \pm 0.67 \mathrm{~mm}$ for the media-adventitia interface, whereas it is $1.24 \pm 0.40 \mathrm{~mm}$ in this study). Also, let us mention that in the study [8], reported in Table I, the segmentation errors are reported in pixels rather than millimeters. No quantitative comparison can thus be made. On the other hand, in the study [34], the maximum and mean standard deviations for manual segmentations of the carotid surface were 1.64 and $0.45 \mathrm{~mm}$, respectively. We interpret those two measures as the Hausdorff and the mean point-to-point distances between various manual segmentations and their mean. Based on that intraobserver variability study, we conclude that the Hausdorff and mean point-to-point distances of 1.24 and $0.24 \mathrm{~mm}$, respectively, obtained in this study can be considered as good, especially if one considers our large database.

\section{B. Assessment of the Optical Flow Hypothesis}

If our hypotheses on the correlation of the B-mode images and the conservation of their MGL from one frame to the next are true, then the method of Section III-B for estimating the motion field $\tau+\mathcal{L}$ (based on the optical flow identity) makes sense, and we should find a good correlation between two successive frames (taking into account the motion field), and a ratio of their MGL near 1 in our database. 
For each of the 130 video-sequence segmentations, we estimated the motion field as in Section III-B, on a neighborhood of the segmented region at each frame $t$ of the video sequence. We then computed, for each frame, the correlation coefficient and the MGL ratio between the images at that frame and the next frame, as well as the Euclidean norm of the deformation matrix of the estimated motion field between the two frames. We then took the average values of these three measures over the 130 video sequences of the database. The correlation coefficient was $0.97 \pm 0.031$, the MGL ratio was $1.0 \pm 0.0024$, and the Euclidean norm of the deformation matrix was $0.012 \pm 0.0068$, which means that the deformation $\mathcal{L}$ of (1) was near the identity operator. In our opinion, this indicates that the use of optical flow was self-consistent in the context of this application.

\section{Evaluation of the Overall Movement of the Plaque}

The results of Section VII-B indicate that the use of the optical flow method was reasonable to perform the tracking of the plaque over the video sequence. But, was tracking of the plaque necessary? To answer this question, we considered for each of the 130 segmented video sequences, the geometric center of the segmented plaque $\left(x_{c, t}, y_{c, t}\right)$ at frame $t=1, \ldots, T$. The curves $\left(x_{c, t}\right)_{t=1}^{T}$ and $\left(y_{c, t}\right)_{t=1}^{T}$ yield the tracking of the geometric center of the plaque over the video sequence in the lateral and axial directions. The measures $D x=\max \left(x_{c, t}\right)-\min \left(x_{c, t}\right)$ and $D y=\max \left(y_{c, t}\right)-\min \left(y_{c, t}\right)$ give the total lateral and axial displacements. We obtained $D x=1.07 \pm 0.49 \mathrm{~mm}$ and $D y=0.52 \pm 0.30 \mathrm{~mm}$. We conclude that tracking of the plaque was necessary.

\section{Strengths and Limitations of the Method}

The average computation time was $38 \mathrm{~s}$ per frame. Further efficiency can be made on a commercial version, provided one is given a few CPUs combined with a GPU implementation. In fact, the EM and the ES algorithms would benefit from parallelization. So, the clinician might carry on the patient examination, while the segmentation of the whole sequence is performed. Thus, there is a reasonable hope to see a clinically applicable version in the future. On the other hand, we evaluated that the expert technician spent about $9.6 \mathrm{~s}$ per frame for the manual segmentations. An average processing time of $20 \mathrm{~s}$ per image is reported in [8, p. 1267, end of Section II].

The proposed method is semiautomatic, in the sense that it requires an initial manual segmentation of the plaque in the first frame, and then proceeds to the segmentation of the entire sequence without further user interaction. In particular, the method depends on the initial segmentation. We performed segmentations on the entire database following a random perturbation of the manual segmentation in the first frame within a distance of $0.5 \mathrm{~mm}$. Note that the mean intraobserver variability reported in [34] is $0.45 \mathrm{~mm}$. Paired $t$-tests with a confidence level of 0.05 showed that there were no significant difference in the eight evaluation measures used (based on the manual segmentations without random perturbation). Thus, the method is robust to a reasonable variability in the initialization. On the other hand, it is understood that the initial manual segmentation is user de- pendent, and in fact, corresponds to prior information on the identification of the carotid artery within the image, the detection, and the localization of a plaque.

In the plaque segmentation methods of [6], [8] and [10], the initialization of the algorithm was performed automatically, based on blood flow, echogenicity of the lumen and the plaque, or the Hough transform, respectively. Moreover, none of these methods was presented nor tested in the context of a sequence of ultrasound images. Now, in principle, if a method works well for an individual image, it might work well for a whole sequence of images, each one treated individually. However, we are inclined to think that it is preferable to perform the motion tracking over the sequence, based on a plaque segmentation in a reference frame (for instance, the first one). Then, the plaque segmentation in the reference frame might be done automatically with one of the methods [6], [8], [10].

We performed one-way analyses of variance on the eight evaluation measures of the segmentation algorithm among the groups determined by the degree of stenosis or calcification, and there were no statistically significant differences, except for the kappa index and the overlap. However, a pairwise comparison gave significant differences for the kappa index and the overlap only between mild and moderate calcification (and not between no calcification and severe calcification). Thus, the performance of the algorithm is not inversely proportional to the severity of stenosis or calcification. The evaluation measures of the algorithm according to the degree of stenosis or calcification are presented in Table V. Incidentally, Fig. 1 is an example of a plaque with calcification.

In the studies [6], [8] and [10], the US images were B-mode scans with further postprocessing, such as speckle reduction filtering [6] or low-pass filtering [8], [10]. Since the segmentation methods in these studies are mainly based on gradients, a postprocessing step was desirable. In this study, the proposed segmentation method is based on the statistical distribution of the gray level in the echo envelope of the RF signal. Since it is a region-based segmentation method, we believe that it is less likely to be sensitive to the image quality and image noise than a method based solely on gradients, that is to say without postprocessing steps, but we have not tested this aspect. Note that Nakagami distributions, viewed as approximations of homodyned K-distributions, are well suited to model the echo envelope of RF images [35]. On the other hand, if the B-mode image is postprocessed, one may consider gamma distributions, rather than Nakagami distributions, to model the gray level of B-mode images ${ }^{2}$. See [36] for instance. In particular, with that slight modification in mind, the proposed method may also be applied to B-mode scans with manufacturer proprietary postprocessing.

The proposed method is not meant as a registration method in the context of large motion as it stands. Such a context would require further development of the method.

\footnotetext{
${ }^{2}$ One should not confuse the gamma distribution on the gray level of the B-mode image with the gamma distribution on the intensity of the B-mode image (i.e., the square of the gray level). The latter is equivalent to a Nakagami distribution on the gray level itself, as mentioned in Section IV-B.
} 
TABLE V

Eight Evaluation MeAsures of the Algorithm ACCORDing to the DEGREE OF StenOSIS OR CALCIFICATION

\begin{tabular}{|c|c|c|c|c|c|c|c|c|}
\hline & \multicolumn{8}{|c|}{ Evaluation measures } \\
\hline Stenosis & se $(\%)$ & $\mathrm{sp}(\%)$ & KI (\%) & ov $(\%)$ & $\begin{array}{c}\text { EArea }(\%) \\
\text { (plaque) }\end{array}$ & $\begin{array}{c}\text { AEArea }(\%) \\
\text { (plaque) }\end{array}$ & $\mathrm{D}(\mathrm{mm})$ & $\mathrm{HD}(\mathrm{mm})$ \\
\hline $0-30 \%$ & $83.7 \pm 6.5$ & $93.5 \pm 4.6$ & $84.5 \pm 5.8$ & $73.9 \pm 8.3$ & $-2.04 \pm 7.46$ & $6.90 \pm 4.18$ & $0.22 \pm 0.07$ & $1.21 \pm 0.43$ \\
\hline $30-50 \%$ & $78.9 \pm 9.5$ & $93.7 \pm 4.6$ & $81.1 \pm 8.3$ & $69.5 \pm 10.7$ & $-5.81 \pm 7.08$ & $7.63 \pm 5.60$ & $0.28 \pm 0.09$ & $1.21 \pm 0.37$ \\
\hline $50-70 \%$ & $85.1 \pm 8.4$ & $95.5 \pm 2.0$ & $86.7 \pm 7.3$ & $77.3 \pm 10.5$ & $-3.84 \pm 6.97$ & $6.36 \pm 5.25$ & $0.23 \pm 0.07$ & $1.16 \pm 0.37$ \\
\hline$>70 \%$ & $85.0 \pm 8.9$ & $93.7 \pm 4.8$ & $85.3 \pm 8.5$ & $75.5 \pm 10.7$ & $-0.48 \pm 9.26$ & $7.68 \pm 5.31$ & $0.26 \pm 0.10$ & $1.35 \pm 0.40$ \\
\hline \multicolumn{9}{|l|}{ Calcification } \\
\hline none & $82.6 \pm 8.7$ & $94.6 \pm 4.2$ & $83.0 \pm 7.4$ & $71.9 \pm 9.8$ & $-1.08 \pm 9.95$ & $8.53 \pm 5.38$ & $0.21 \pm 0.08$ & $1.11 \pm 0.33$ \\
\hline mild & $80.5 \pm 9.2$ & $92.2 \pm 6.3$ & $81.5 \pm 9.2$ & $70.2 \pm 11.3$ & $-2.39 \pm 8.82$ & $7.92 \pm 5.03$ & $0.25 \pm 0.09$ & $1.27 \pm 0.47$ \\
\hline moderate & $86.1 \pm 6.5$ & $95.0 \pm 2.4$ & $87.5 \pm 5.2$ & $78.4 \pm 7.7$ & $-3.42 \pm 6.71$ & $6.11 \pm 4.81$ & $0.24 \pm 0.07$ & $1.26 \pm 0.37$ \\
\hline severe & $83.1 \pm 8.9$ & $94.0 \pm 3.1$ & $84.3 \pm 7.2$ & $73.7 \pm 9.7$ & $-3.06 \pm 7.84$ & $7.22 \pm 4.63$ & $0.28 \pm 0.10$ & $1.31 \pm 0.44$ \\
\hline
\end{tabular}

The sensitivity (se), the specificity (sp), the kappa index (KI), the overlap (ov), the error of area (EA rea), the absolute error of area (AEArea), the mean point-to-point distance $(D)$, and the Hausdorff distance (HD).

\section{CONCLUSION}

We have included a prediction of the segmented plaque, based on motion field estimates, into the prior of a Bayesian model, in the form of a spatiotemporal cohesion constraint. The computation of the MAP of that Bayesian model then allowed the refinement of the prediction. Our tests showed that tracking of the plaque is necessary, and that motion field estimates based on the optical flow identity is self-consistent in the context of our database. The motion fields were modeled by a first-order tissue dynamics. In addition to the spatiotemporal cohesion constraint, the prior of the segmentation model included a smoothing constraint and we proposed a method for estimating the weight of that smoothing constraint. The likelihood of the Bayesian model was based on mixtures of Nakagami distributions that are well suited to model complex echogenicity observed in the B-mode envelope of RF acquisitions. In that model, each of the tissues (lumen, plaque, adventitia) corresponded to a weighted sum of these Nakagami distributions that could be learned from the solution at the previous frame. This flexibility of the model took into account the variability of appearance of the tissues within sequences, and did not require any off-line learning step. The proposed method requires only the manual segmentation of the plaque in the first frame of the video sequence. Our tests indicate that the semiautomatic segmentations of plaques located at internal proximal carotid arteries obtained by the proposed method compare favorably to the state-of-the-art segmentation method [6] (see Table III). Finally, we think that an optimized implementation based on parallelization could provide close to real-time processing.

\section{APPENDIX}

To estimate the weight $\beta>0$ of the geometrical smoothness constraint $\Delta_{\mathrm{sm}, d}$ appearing in (11), we considered the Markov point process having the following likelihood:

$$
\begin{aligned}
& P\left(\left(\gamma_{1}, \gamma_{2}\right) \mid \beta\right) \\
& \quad=\frac{\exp \left(-\beta\left\{\Delta_{\mathrm{sm}, d}\left(\gamma_{1}\right)+\Delta_{\mathrm{sm}, d}\left(\gamma_{2}\right)\right\}\right)}{\iint \exp \left(-\beta\left\{\Delta_{\mathrm{sm}, d}\left(\gamma_{1}\right)+\Delta_{\mathrm{sm}, d}\left(\gamma_{2}\right)\right\}\right) d \gamma_{1} d \gamma_{2}}
\end{aligned}
$$

where $\gamma_{1}$ and $\gamma_{2}$ represent two continuous piecewise linear curves. The denominator is called the partition function and is analytically and numerically intractable, thus making the maximization of (17) as a function of $\beta$ practically impossible.

Let $\left(\left(x_{l}, y_{l}\right)\right)_{l=1}^{L_{1}}$ and $\left(\left(\tilde{x}_{l}, \tilde{y}_{l}\right)\right)_{l=1}^{L_{2}}$ be the sequences of the vertices of the curves $\gamma_{1}$ and $\gamma_{2}$, respectively. For $l=1, \ldots, L_{1}$, $\gamma_{1} \backslash\left(x_{l}, y_{l}\right)$ denotes the curve obtained by deleting the point $\left(x_{l}, y_{l}\right)$, whereas $\gamma_{1} \backslash\left(x_{l}, y_{l}\right) \cup\left(\xi_{l}, \eta_{l}\right)$ denotes the curve obtained by replacing $\left(x_{l}, y_{l}\right)$ with $\left(\xi_{l}, \eta_{l}\right)$. The analogous notation applies to $\gamma_{2}$. In [29], the pseudolikelihood $P_{\mathrm{PL}}\left(\left(\gamma_{1}, \gamma_{2}\right) \mid \beta\right)$ of a Markov model is proposed as an alternative to its likelihood:

$$
\begin{aligned}
& \prod_{l=1}^{L_{1}} \frac{\exp \left(-\beta \Delta_{\mathrm{sm}, d}\left(\gamma_{1}\right)\right)}{\iint \exp \left(-\beta \Delta_{\mathrm{sm}, d}\left(\gamma_{1} \backslash\left(x_{l}, y_{l}\right) \cup\left(\xi_{l}, \eta_{l}\right)\right)\right) d \xi_{l} d \eta_{l}} \\
& \quad \times \prod_{l=1}^{L_{2}} \frac{\exp \left(-\beta \Delta_{\mathrm{sm}, d}\left(\gamma_{2}\right)\right)}{\iint \exp \left(-\beta \Delta_{\mathrm{sm}, d}\left(\gamma_{2} \backslash\left(\tilde{x}_{l}, \tilde{y}_{l}\right) \cup\left(\tilde{\xi}_{l}, \tilde{\eta}_{l}\right)\right)\right) d \tilde{\xi}_{l} d \tilde{\eta}_{l}} .
\end{aligned}
$$

In order to handle the degenerate case, where the maximum of the pseudolikelihood is reached at infinity (corresponding to $\Delta_{\mathrm{sm}, d}\left(\gamma_{1}\right)+\Delta_{\mathrm{sm}, d}\left(\gamma_{2}\right)=0$ ), we found convenient to consider a prior on the hyperparameter $\beta$ of the form $P(\beta)=\mathcal{G}(\beta \mid k, \theta)$, with $k>1$ and $\theta>0$. Indeed, in that case, the function $\frac{d \log }{d \beta} P_{\mathrm{PL}}\left(\left(\gamma_{1}, \gamma_{2}\right) \mid \beta\right)+\frac{d \log }{d \beta} P(\beta)$ has a unique root and it corresponds to the maximum of the function $P_{\mathrm{PL}}\left(\left(\gamma_{1}, \gamma_{2}\right) \mid \beta\right) P(\beta)$.

In our tests, we took systematically $k=2$ and $\theta=1$, so that the distribution $P(\beta)$ has the mean $k \theta=2$ and variance $k \theta^{2}=2$. This yields a median of 1.68 , a $2.5 \%$ percentile of 0.24 , and a $97.5 \%$ percentile of 5.57 . With this prior, the estimated value of $\beta$ varied between 0.2 and 10 , in our tests. In particular, the estimation of $\beta$ was worthwhile.

\section{ACKNOWLEDGMENT}

Dr. Soulez is recipient of a National Scientist award and Dr. Cloutier was recipient of the same award of the Fonds de la Recherche en Santé du Québec. Dr. Destrempes received an additional scholarship from the Groupe de Recherche en Sciences et Technologies Biomédicales of the Institut de Génie Biomédical, Université de Montréal. The authors are grateful to S. Fortin for technical assistance in performing the manual segmentations and to É. Mercure for supervising her work with 
Dr. G. Soulez. The authors thank the anonymous reviewers for their comments.

\section{REFERENCES}

[1] C. Schmitt, G. Soulez, R. L. Maurice, M.-F. Giroux, and G. Cloutier, "Noninvasive vascular elastography: Toward a complementary characterization tool of atherosclerosis in carotid arteries," Ultrasound Med. Biol., vol. 33 , no. 12, pp. 1841-1858, 2007.

[2] H. Ribbers, R. G. P. Lopata, S. Holewijn, G. Pasterkamp, J. D. BlankenSteijn, and C. L. de Korte, "Noninvasive two-dimensional strain imaging of arteries: Validation in phantoms and preliminary experience in carotid arteries in vivo," Ultrasound Med. Biol., vol. 33, no. 4, pp. 530540, 2007.

[3] H. Shi, C. C. Mitchell, M. McCormick, M. A. Kliewer, R. J. Dempsey, and T. Varghese, "Preliminary in vivo atherosclerotic carotid plaque characterization using the accumulated axial strain and relative lateral shift strain indices," Phys. Med. Biol., vol. 53, pp. 6377-6394, 2008.

[4] T. Yamagishi, M. Kato, Y. Koiwa, K. Omata, H. Hasegawa, and H. Kanai, "Evaluation of plaque stabilization by fluvastatin with carotid intimamedial elasticity measured by a transcutaneous ultrasonic-based tissue characterization system," J. Atherosclerosis Thrombosis, vol. 16, no. 5, pp. 662-673, 2009.

[5] C. P. Loizou, C. S. Pattichis, R. S. H. Istepanian, M. Pantziaris, and A. Nicolaides, "Atherosclerotic carotid plaque segmentation," in Proc. 29th Ann. Int. Conf. IEEE Engineering in Medicine and Biology Society (EMBS), San Francisco, CA, Sep. 1-5, 2004, pp. 1403-1406.

[6] C. P. Loizou, C. S. Pattichis, M. Pantziaris, and A. Nicolaides, "An integrated system for the segmentation of atherosclerosis carotid plaque," IEEE Trans. Inform. Technol. Biomed., vol. 11, no. 6, pp. 661-667, Nov. 2007.

[7] S. Delsanto, F. Molinari, W. Liboni, P. Giustetto, S. Badalamenti, and J. S. Suri, "User-independent plaque characterization and accurate IMT measurement of carotid artery wall using ultrasound," in Proc. 28th Ann. Int. Conf. IEEE Engineering in Medicine and Biology Society (EMBS), New York City, NY, Aug. 30-Sep. 3, 2006, pp. 2404-2407.

[8] S. Delsanto, F. Molinari, P. Giustetto, W. Liboni, S. Badalamenti, and J. S. Suri, "Characterization of a completely user-independent algorithm for carotid artery segmentation in 2-D ultrasound images," IEEE Trans. Instr. Meas., vol. 56, no. 4, pp. 1265-1274, Aug. 2007.

[9] F. Molinari, W. Liboni, E. Pavanelli, P. Giustetto, S. Badalamenti, and J. S. Suri, "Accurate and automatic carotid plaque characterization in contrast enhanced 2-D ultrasound images," in Proc. 29th Ann. Int. Conf. IEEE Engineering in Medicine and Biology Society (EMBS), Cité Internationale, Lyon, France, Aug. 23-26, 2007, pp. 335-338.

[10] S. Golemati, J. Stoitsis, E. G. Sifakis, T. Balkisas, and K. S. Nikita, "Using the Hough transform to segment ultrasound images of longitudinal and transverse sections of the carotid artery," Ultrasound Med. Biol., vol. 33, no. 12, pp. 1918-1932, 2007.

[11] P. Abolmaesumi, M. R. Sirouspour, and S. E. Salcudean, "Real-time extraction of carotid artery contours from ultrasound images," in Proc. 13th IEEE Symp. Computer-Based Medical Systems (CBMS), Houston, TX, Jun. 2000, pp. 181-186.

[12] J. Guerrero, S. E. Salcudean, J. A. McEwen, B. A. Masri, and S. Nicolaou, "Real-time vessel segmentation and tracking for ultrasound imaging applications," IEEE Trans. Med. Imaging, vol. 26, no. 8, pp. 1079-1090, Aug. 2007.

[13] J. D. Gill, H. M. Ladak, D. A. Steinman, and A. Fenster, "Segmentation of ulcerated plaque: A semi-automatic method for tracking the progression of carotid atherosclerosis," in Proc. World Congr. Med. Phys. Biomed. Eng., Chicago, IL, 2000, pp. 1-4.

[14] F. Mao, J. Gill, D. Downey, and A. Fenster, "Segmentation of carotid artery in ultrasound images: Method development and evaluation technique," Med. Phys., vol. 27, no. 8, pp. 1-10, 2000.

[15] G. Slabaugh, G. Unal, M. Wels, T. Fang, and B. Rao, "Statistical regionbased segmentation of ultrasound images," Ultrasound Med. Biol., vol. 35, no. 5, pp. 781-795, 2009.

[16] J. A. Noble and D. Boukerroui, "Ultrasound image segmentation: A survey," IEEE Trans. Med. Imaging, vol. 25, no. 8, pp. 987-1010, Aug. 2006.

[17] J. A. Noble, "Ultrasound image segmentation and tissue characterization," Proc. Institution Mech. Eng., Part H: J. Eng. Med., vol. 224, no. 2, pp. 307316, 2010.
[18] E. Kyriacou, C. Pattichis, M. Pattichis, C. Loizou, C. Christodoulou, S. Kakkos, and A. Nicolaides, "A review of noninvasive ultrasound image processing methods in the analysis of carotid plaque morphology for the assessment of stroke risk," IEEE Trans. Inform. Technol. Biomed., vol. 14, no. 4, pp. 1027-1038, 2010.

[19] F. Destrempes, J. Meunier, M.-F. Giroux, G. Soulez, and G. Cloutier, "Segmentation in ultrasonic B-mode images of healthy carotid arteries using mixtures of Nakagami distributions and stochastic optimization," IEEE Trans. Med. Imaging, vol. 28, no. 2, pp. 215-229, Feb. 2009.

[20] F. Destrempes, G. Soulez, M.-F. Giroux, J. Meunier, and G. Cloutier, "Segmentation of plaques in sequences of ultrasonic B-mode images of carotid arteries based on motion estimation and Nakagami distributions," in Proc. IEEE Int. Ultrasonics Symp., Rome, Italy, Sep. 2009, pp. 2480 2483.

[21] I. Mikic, S. Krucinski, and J. D. Thomas, "Segmentation and tracking in echocardiographic sequences: Active contours guided by optical flow estimates," IEEE Trans. Med. Imaging, vol. 17, no. 2, pp. 274-284, Apr. 1998.

[22] E. G. Grant, C. B. Benson, G. L. Moneta, A. V. Alexandrov, J. D. Baker, E. I. Bluth, B. A. Carroll, M. Eliasziw, G. Gocke, B. S. Hertzberg, S. Katanick, L. Needleman, J. Pellerito, J. F. Polak, K. S. Rholl, D. L. Wooster, and E. Zierler, "Carotid artery stenosis: Grayscale and Doppler US diagnosis: Society of Radiologists in Ultrasound Consensus Conference," Radiology, vol. 229, no. 2, pp. 340-346, 2003.

[23] NASCET Collaborators, "Beneficial effect of carotid endarterectomy in symptomatic patients with high-grade carotid stenosis," N. Engl. J. Med., vol. 325, pp. 445-453, 1991.

[24] J. Meunier and M. Bertrand, "Ultrasonic texture motion analysis: Theory and simulation," IEEE Trans. Med. Imaging, vol. 14, no. 2, pp. 293-300, Jun. 1995.

[25] R. L. Maurice, J. Ohayon, Y. Frétigny, M. Bertrand, G. Soulez, and G. Cloutier, "Non-invasive vascular elastography: Theoretical framework," IEEE Trans. Med. Imaging, vol. 23, no. 2, pp. 164-180, Feb. 2004.

[26] M. Cinthio and R. Ahlgren, "Intra-observer variability of longitudinal displacement and intramural shear strain measurements of the arterial wall using ultrasound noninvasively in vivo," Ultrasound Med. Biol., vol. 36, no. 5, pp. 697-704, 2010.

[27] A. Dempster, N. Laird, and D. Rubin, "Maximum likelihood from incomplete data via the EM algorithm," J. Roy. Stat. Soc. (Ser. B), vol. 39, pp. 1-38, 1977.

[28] J. M. Meinders, L. Kornet, and A. P. G. Hoeks, "Assessment of spatia inhomogeneities in intima media thickness along an arterial segment using its dynamic behavior," Am. J. Physiol.: Heart Circulatory Physiol., vol. 285, pp. H384-H391, 2003.

[29] J. Besag, "Statistical analysis of non-lattice data," Statistician, vol. 24, no. 3, pp. 179-195, 1975.

[30] F. Comets, "On consistency of a class of estimators for exponential families of Markov random fields on the lattice," Ann. Stat., vol. 20, no. 1, pp. 455468, 1992.

[31] C. Metz, "Basic principles of ROC analysis," Semin. Nucl. Med., vol. 8, pp. 283-298, 1978.

[32] T. Fawcett, "An introduction to ROC analysis," Pattern Recognit. Lett., vol. 27, pp. 861-874, 2006.

[33] D. P. Huttenlocher, G. A. Klanderman, and W. J. Rucklidge, "Comparing images using the Hausdorff distance," IEEE Trans. Pattern Anal. Mach. Intell., vol. 15, pp. 850-863, Sep. 1993.

[34] J. D. Gill, H. M. Ladak, D. A. Steinman, and A. Fenster, "Accuracy and variability assessment of a semiautomatic technique for segmentation of the carotid arteries from three-dimensional ultrasound images," Med. Phys., vol. 27, no. 6, pp. 1333-1342, 2000.

[35] F. Destrempes and G. Cloutier, "A critical review and uniformized representation of statistical distributions modeling the ultrasound echo envelope," Ultrasound Med. Biol., vol. 36, no. 7, pp. 1037-1051, 2010.

[36] Z. Tao, H. D. Tagare, and J. D. Beaty, "Evaluation of four probability distribution models for speckle in clinical cardiac ultrasound images," IEEE Trans. Med. Imaging, vol. 25, no. 11, pp. 1483-1491, Nov. 2006.

Authors' photographs and biographies not available at the time of publication. 